\title{
Parametric Sensitivity Analysis for Importance Measure on Failure Probability and Its Efficient Kriging Solution
}

\author{
Yishang Zhang, Yongshou Liu, and Xufeng Yang \\ Institute of Aircraft Reliability Engineering, Department of Engineering Mechanics, Northwestern Polytechnical University, \\ Xian 710129, China \\ Correspondence should be addressed to Yishang Zhang; cloth1169@163.com
}

Received 27 May 2014; Revised 23 September 2014; Accepted 23 September 2014

Academic Editor: Shaomin Wu

Copyright (c) 2015 Yishang Zhang et al. This is an open access article distributed under the Creative Commons Attribution License, which permits unrestricted use, distribution, and reproduction in any medium, provided the original work is properly cited.

The moment-independent importance measure (IM) on the failure probability is important in system reliability engineering, and it is always influenced by the distribution parameters of inputs. For the purpose of identifying the influential distribution parameters, the parametric sensitivity of IM on the failure probability based on local and global sensitivity analysis technology is proposed. Then the definitions of the parametric sensitivities of IM on the failure probability are given, and their computational formulae are derived. The parametric sensitivity finds out how the IM can be changed by varying the distribution parameters, which provides an important reference to improve or modify the reliability properties. When the sensitivity indicator is larger, the basic distribution parameter becomes more important to the IM. Meanwhile, for the issue that the computational effort of the IM and its parametric sensitivity is usually too expensive, an active learning Kriging (ALK) solution is established in this study. Two numerical examples and two engineering examples are examined to demonstrate the significance of the proposed parametric sensitivity index, as well as the efficiency and precision of the calculation method.

\section{Introduction}

Uncertainties existing in engineering analysis and design are inherently unavoidable in nature associated with the manufacturing error, material property, loads, and so forth. Fortunately, reliability analysis and sensitivity analysis are now available to deal with the uncertainty existing in design variables to improve the performance of a mechanical or structural system [1-3]. Reliability analysis aims at predicting the failure probability (or reliability) of the structure under the effects of random uncertainties. On the other hand, sensitivity analysis focuses on the contribution of each uncertainty or distribution parameters of the input variables [4-7]. It is reasonable and practicable to obtain reliability sensitivity analysis for quantifying and ranking the effects of random uncertainties on the failure probability. In this paper, the reliability sensitivity analysis is concerned.

Sensitivity analysis (SA) is the study of how the output response of a model (numerical or otherwise) is affected by the input uncertainty, which can be classified into two groups: local SA and global SA [8]. The local SA investigates how small variation of the distribution parameters near a reference point changes the output value. The classical local SA is defined as the partial derivative of the output with respect to the distribution parameters of inputs [9]. The global SA, also named as importance measure (IM), gives consideration to measure the effect of the output uncertainty on the uncertainty of the input parameters, covering their variation range space as opposed to local SA using partial derivatives.

Saltelli and Marivoet [10] and Helton and Davis [11] proposed the nonparametric techniques (input-output correlation), but this method lacks model independence. With the advantage of "global, quantitative and model free," the variance-based importance measures are gaining the increasing attention of practitioners and have been used extensively for quantitative analysis [12-16]. However, Borgonovo addressed the following fact: "the premise of variance-based GSA technique that the variance is sufficient to identify the variability of model output is not always true" [17]. The "moment-independent" importance measures have been presented [6, 16-19]. They are also global, quantitative, 
model-free, and additionally moment-independent, thus attracting more and more attention of practitioners recently. Generally speaking, in reliability analysis, researchers often pay the most attention to the failure probability. With this respect, Cui et al. [6] introduced a moment-independent importance measure of the basic variable on the failure probability which was further developed by Li et al. [20].

This moment-independent importance measure on failure probability is applied to quantify the average effect of the basic variables on the reliability of the model and obtain the importance ranking. The IM on the failure probability can be used in the priordesign stage for variables screening when a reliability design solution is yet identified and the postdesign stage for uncertainty reduction after an optimal design has been determined. Uncertain inputs inherent in most engineering problems are assumed as random variables obeying probabilistic distributions. Obviously, system reliability and reliability IM on failure probability are decided by distribution parameters. One can directly change the input's IMs by controlling or modifying some input's distribution parameters; namely, changing the input's distribution parameters can also influence the failure probability, which would facilitate its use under various scenarios of design under uncertainty, for instance, in reliability-based design. It is necessary to further recognize effects of the distribution parameters within system reliability on the importance ranking. At present, Cui et al. [7] defined the parametric sensitivities to illustrate the influences of the distribution parameters on the importance measures.

Combined with the local SA technique of input parameters, the effects of the distribution parameters on the IM on failure probability can be introduced, by which IMs of the inputs can be controlled or modified by changing the distribution parameters. This can provide important guidance for robust design, reliability-based design, and reliability-based optimization in engineering. However, its solution still relies on the corresponding method for failure probability and the computation of the derivative operation on failure probability existing in the parametric sensitivity of IM.

The Monte Carlo simulation (MCS) procedure is easy to implement and is available for computing the parametric sensitivity of IM based purely on model evaluation $[15,21]$, but it has to face the problem of "curse of computational cost" for the problem with small failure probability $\left(10^{-3}\right.$ $10^{-4}$ or smaller). Thus, to deal with this problem, the Kriging approach is widely used for deterministic optimization problems [22] and reliability analysis [23] has been intensively investigated. Furthermore, an advanced Kriging method, named active learning Kriging (ALK), has been proved to be highly efficient in reliability analysis problems [24-26]. This work would employ the ALK method to compute the parametric sensitivity of IM on failure probability. In the ALK method, the Kriging model is updated by adding new training points to the design of experiment (DOE) in iterations by active learning until the Kriging model satisfies necessary accuracy. The computational efficiency of the Kriging method can be validated by several numerical and engineering examples.
The remainder of this work is organized as follows. Section 2 reviews the definition of the moment-independent importance measure of the basic variable on the failure probability. And the parametric sensitivity of IM on failure probability is firstly presented. In Section 3, the established ALK solution can effectively solve the problem that the computational cost of the parametric sensitivity of IM relies on small failure probability. The effectiveness of the proposed parametric sensitivity of IMs and efficiency of the ALK method are demonstrated by several examples in Section 4 . The discussions and conclusions are given at the end of this paper.

\section{Definition of the Parametric Sensitivity of IM on Failure Probability}

2.1. Review of the Importance Measure on the Failure Probability. Consider a probabilistic reliability model $Y=$ $g\left(X_{1}, X_{2} \cdots X_{n}\right)$, where $g\left(X_{1}, X_{2} \cdots X_{n}\right)$ is the performance function, $Y$ is the model output, and $\mathbf{X}=\left\{X_{1}, X_{2} \cdots X_{n}\right\}$ is the $n$-dimensional vector of random input variables with joint probability density function (PDF) $f_{\mathbf{X}}(\mathbf{x})$. Denote $P_{f_{Y}}$ by the unconditional failure probability; that is, $P_{f_{Y}}=P\{g(X) \leq 0\}$. When the $i$ th input variable $X_{i}$ is fixed at one given value, the conditional failure probability $P_{f_{Y \mid X_{i}}}$ can be obtained.

Based on the idea of the moment-independent importance analysis, the importance measure of basic variable $\mathbf{X}_{i}$ on the failure probability is defined by Cui et al. [6] as

$$
\begin{aligned}
\eta_{\mathbf{X}_{i}} & =\frac{1}{2} E_{\mathbf{X}_{i}}\left[\left|P_{f_{Y}}-P_{f_{Y \mid \mathbf{X}_{i}}}\right|\right] \\
& =\frac{1}{2} \int_{-\infty}^{+\infty}\left|P_{f_{Y}}-P_{f_{Y \mid \mathbf{X}_{i}}}\right| f_{\mathbf{X}_{i}}\left(\mathbf{x}_{i}\right) d \mathbf{x}_{i}
\end{aligned}
$$

where $\mathbf{X}_{i}$ represents a random basic variable $X_{i}$ or a set of random basic variables $\left(X_{i_{1}}, X_{i_{2}} \cdots X_{i_{g}}\right)$, where $1 \leq i_{1} \leq$ $i_{2} \cdots i_{g} \leq n . E[\cdot]$ is the operator of expectation.

As the absolute value in (1) is difficult to compute, it is transformed into square operation by $\mathrm{Li}$ et al. [20]. The modified version of importance measures on the failure probability can be expressed as follows:

$$
\begin{aligned}
\delta_{\mathbf{X}_{i}}^{P} & =E\left[P_{f_{Y}}-P_{f_{Y \mid \mathbf{x}_{i}}}\right]^{2} \\
& =\int_{-\infty}^{+\infty}\left(P_{f_{Y}}-P_{f_{Y \mid \mathbf{X}_{i}}}\right)^{2} f_{\mathbf{X}_{i}}\left(\mathbf{x}_{i}\right) d \mathbf{x}_{i} .
\end{aligned}
$$

In the reliability analysis, the failure domain of this structure system is defined as

$$
F=(\mathbf{x}: g(\mathbf{x})<0)
$$

Suppose the indicator function of this failure domain is given as $I_{F}(\mathbf{x})$; that is,

$$
I_{F}(\mathbf{x})= \begin{cases}1 & g(\mathbf{x})<0, \\ 0 & g(\mathbf{x})>0 .\end{cases}
$$


Then, the unconditional failure probability and conditional failure probability on $X_{i}$ can be expressed as

$$
\begin{aligned}
P_{f_{Y}} & =\int \cdots \int I_{F}(\mathbf{x}) f_{\mathbf{X}}(\mathbf{x}) d \mathbf{x}=E\left(I_{F}(\mathbf{x})\right), \\
P_{f_{Y \mid X_{i}}} & =\int \cdots \int I_{F}(\mathbf{x}) f_{\mathbf{X}}\left(\mathbf{x} \mid x_{i}\right) d \mathbf{x}=E\left(I_{F}\left(\mathbf{x} \mid x_{i}\right)\right) .
\end{aligned}
$$

Here, $f_{\mathbf{X}}\left(\mathbf{x} \mid x_{i}\right)$ is the conditional joint PDF on $x_{i}$. According to the probability theory, $f_{\mathbf{X}}\left(\mathbf{x} \mid x_{i}\right)$ is defined as

$$
f_{\mathbf{X}}\left(\mathbf{x} \mid x_{i}\right)=\frac{f_{\mathbf{X}}(\mathbf{x})}{f_{X_{i}}\left(x_{i}\right)}
$$

where $f_{X_{i}}\left(x_{i}\right)$ is the PDF of $x_{i}$.

2.2. The Parametric SA of IM on Failure Probability. For the influential distribution parameter, it is significant to identify how it influences IM on failure probability. We suppose that each input only depends on one distribution parameter in order to simplify the notation in the following.

As stated above, $\theta_{X_{i}}^{j}(i=1,2 \cdots n)$ is the $j$ th distribution parameter of input $X_{i}$ which influences the unconditional failure probability $P_{f_{Y}}$, but not the conditional failure probability $P_{f_{Y \mid X_{i}}}$. It is also noticed that $\theta_{X_{k}}^{j} \in \boldsymbol{\theta}_{-X_{i}}^{j}$ is not the distribution parameter of input $X_{i}$, but it still influences $P_{f_{Y}}$ and $P_{f_{Y \mid X_{i}}} \cdot \boldsymbol{\theta}_{-X_{i}}$ is a vector containing all distribution parameters of the input variables but $X_{i}$. The contributions of distribution parameter $\theta_{X_{i}}^{j}$ on $P_{f_{Y}}$ and $P_{f_{Y \mid X_{i}}}$ can be shown in Figure 1.

To analyze the effect of changing the $j$ th parameter $\theta_{X_{i}}^{j}(i=1,2 \cdots n)$ of input $X_{i}$, the sensitivity derivative of the IM on failure probability $\delta_{\mathbf{X}_{i}}^{P}$ can be defined as

$$
\begin{aligned}
& \frac{\partial \delta_{X_{i}}^{P}}{\partial \theta_{X_{i}}^{j}} \\
& =\frac{\partial \int_{-\infty}^{+\infty}\left(P_{f_{Y}}-P_{f_{Y \mid X_{i}}}\right)^{2} f_{X_{i}}\left(x_{i}\right) d x_{i}}{\partial \theta_{X_{i}}^{j}} \\
& =\int_{-\infty}^{+\infty}\left[2\left(P_{f_{Y}}-P_{f_{Y \mid X_{i}}}\right) \cdot \frac{\partial P_{f_{Y}}}{\partial \theta_{X_{i}}^{j}}\right. \\
& \left.\quad+\left(P_{f_{Y}}-P_{f_{Y \mid X_{i}}}\right)^{2} \frac{\partial f_{X_{i}}\left(x_{i}\right)}{\partial \theta_{X_{i}}^{j}} \frac{1}{f_{X_{i}}\left(x_{i}\right)}\right] f_{X_{i}}\left(x_{i}\right) d x_{i} .
\end{aligned}
$$

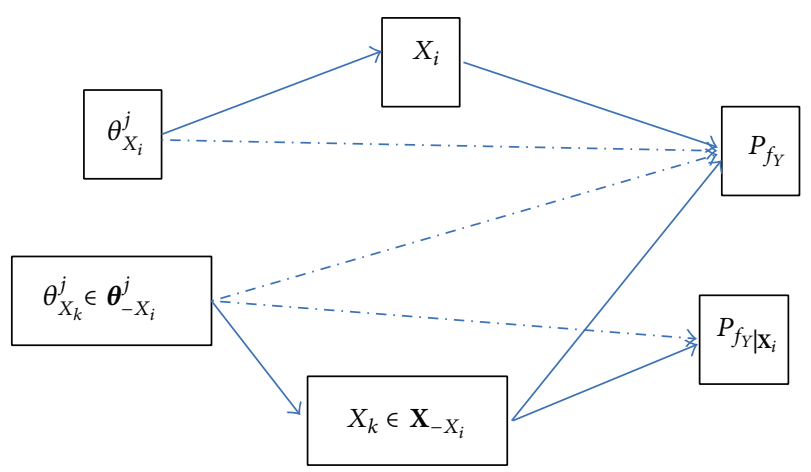

FIGURE 1: Influence of the distribution parameter on the $P_{f_{Y}}$ and $P_{f_{Y \mid \mathbf{X}_{i}}}$.

To analyze the effect of changing the $j$ th parameter $\theta_{X_{k}}^{j} \epsilon$ $\boldsymbol{\theta}_{-X_{i}}^{j}$ of input on $\delta_{\mathbf{X}_{i}}^{P}$, the derivative of the IM can be defined as

$$
\begin{aligned}
& \frac{\partial \delta_{X_{i}}^{P}}{\partial \theta_{X_{k}}^{j}} \\
& =\frac{\partial \int_{-\infty}^{+\infty}\left(P_{f_{Y}}-P_{f_{Y \mid X_{i}}}\right)^{2} f_{X_{i}}\left(x_{i}\right) d x_{i}}{\partial \theta_{X_{k}}^{j}} \\
& =\int_{-\infty}^{+\infty}\left[2\left(P_{f_{Y}}-P_{f_{Y \mid X_{i}}}\right) \cdot\left(\frac{\partial P_{f_{Y}}}{\partial \theta_{X_{k}}^{j}}-\frac{P_{f_{Y \mid X_{i}}}}{\partial \theta_{X_{k}}^{j}}\right)\right] f_{X_{i}}\left(x_{i}\right) d x_{i} .
\end{aligned}
$$

To compute the above formula, the derivatives of $P_{f_{Y}}$ and $P_{f_{Y \mid X_{i}}}$ contribution with respect to the distribution parameter of input can be given by

$$
\begin{aligned}
\frac{\partial P_{f_{Y}}}{\partial \theta_{X_{i}}^{j}} & =\int \cdots \int_{R^{n}} I_{F}(\mathbf{x}) \frac{\partial f_{\mathbf{X}}(\mathbf{x})}{\partial \theta_{X_{i}}^{j}} \frac{1}{f_{\mathbf{X}}(\mathbf{x})} f_{\mathbf{X}}(\mathbf{x}) d \mathbf{x} \\
& =E\left(\frac{I_{F}(\mathbf{x})}{f_{\mathbf{X}}(\mathbf{x})} \frac{\partial f_{\mathbf{X}}(\mathbf{x})}{\partial \theta_{X_{i}}^{j}}\right) \\
\frac{\partial P_{f_{Y}}}{\partial \theta_{X_{k}}^{j}} & =\int \cdots \int_{R^{n}} I_{F}(\mathbf{x}) \frac{\partial f_{\mathbf{X}}(\mathbf{x})}{\partial \theta_{X_{k}}^{j}} \frac{1}{f_{\mathbf{X}}(\mathbf{x})} f_{\mathbf{X}}(\mathbf{x}) d \mathbf{x} \\
& =E\left(\frac{I_{F}(\mathbf{x})}{f_{\mathbf{X}}(\mathbf{x})} \frac{\partial f_{\mathbf{X}}(\mathbf{x})}{\partial \theta_{X_{k}}^{j}}\right), \\
\frac{P_{f_{Y \mid X_{i}}}}{\partial \theta_{X_{k}}^{j}} & =\int \cdots \int_{R^{n}} I_{F}(\mathbf{x}) \frac{\partial f_{\mathbf{X}}\left(\mathbf{x} \mid x_{i}\right)}{\partial \theta_{X_{k}}^{j}} \frac{1}{f_{\mathbf{X}}\left(\mathbf{x} \mid x_{i}\right)} f_{\mathbf{X}}\left(\mathbf{x} \mid x_{i}\right) d \mathbf{x} \\
& =E\left(\frac{I_{F}(\mathbf{x})}{f_{\mathbf{X}}\left(\mathbf{x} \mid x_{i}\right)} \frac{\partial f_{\mathbf{X}}\left(\mathbf{x} \mid x_{i}\right)}{\partial \theta_{X_{k}}^{j}}\right) .
\end{aligned}
$$


Here, the effects of the distribution parameters on the IMs of input variables can be known, combining local and global sensitivity analysis technology. One can directly optimize the inputs' IMs by controlling or changing some inputs' distribution parameters. It can provide useful information for robust design, reliability-based design, and reliability-based optimization.

In computing the parametric sensitivity of IM, the derivative of the $P_{f_{Y}}$ and $P_{f_{Y \mid x_{i}}}$ with respect to the distribution parameters must be calculated. The performance function expression is characterized by complex implicit limit state functions in most engineering problems, so the derivative of the $P_{f_{Y}}$ and $P_{f_{Y \mid \mathbf{x}_{i}}}$ is without a definite analytic expression. The Monte Carlo simulation (MCS) method can be used for computing the parametric sensitivity of IM. This method has the advantages of high-accuracy and usability based on model evaluation. However, for problems with small failure probability $\left(10^{-3}-10^{-4}\right.$ or smaller), only a very small portion of samples will drop into the failure domain. The computational cost is unacceptable for failure probability and the derivative of the $P_{f_{Y}}$ and $P_{f_{Y \mid X_{i}}}$. For the latter problem, an active learning Kriging method combining MCS is proposed in the next section.

\section{The Active Learning Kriging Method for the Parametric Sensitivity of IM on Failure Probability}

Section 2 tells us that the key point in estimating the global reliability sensitivity indices is to provide the failure probability $P_{f_{Y}}$, the unconditional failure probability $P_{f_{Y \mid X_{i}}}$, and the derivative of $P_{f_{Y}}$ and $P_{f_{Y \mid X_{i}}}$ to the distribution parameters. In practice, there are many engineering problems characterized by complex implicit performance functions, so the computational cost of double-loop MCS method becomes larger especially for finite element (FE) model. To solve this problem, the solution of ALK method is employed to approximate the implicit performance function and improve the efficiency greatly using few training points. Generally speaking, Kriging metamodel is combined by a linear regression component and a stochastic process. It is an interpolation technique based on the statistical theory and has been used to construct the input and output (I/O) systems. The Kriging models are performed with the toolbox DACE, which is a MATLAB toolbox developed by Lophaven et al. [27].

3.1. Kriging Metamodel. The present Kriging model expresses the unknown function $G(\mathbf{x})$ as [22]

$$
G(\mathbf{x})=F(\mathbf{x}, \boldsymbol{\beta})+z(\mathbf{x}),
$$

where $F(\mathbf{x}, \beta)$ is the deterministic part which gives an approximation of the response in mean. The second part $z(x)$ is the realization of stochastic process, which provides the approximation of the local fluctuation so that the whole model interpolates exactly. This part shows the following statistical characteristics:

$$
\begin{aligned}
E[z(x)] & =0, \\
\operatorname{Var}[z(x)] & =\sigma^{2}, \\
\operatorname{Cov}\left[Z\left(x_{i}\right), Z\left(x_{j}\right)\right] & =\sigma^{2}\left[R\left(x_{i}, x_{j}\right)\right] .
\end{aligned}
$$

The mean of this component is zero, the variance is $\sigma^{2}$, and (14) defines its covariance between two points in space. In (15), $x_{i}$ and $x_{j}$ denote two arbitrary points; $R\left(x_{i}, x_{j}\right)$ is the correlation function of $x_{i}$ and $x_{j}$.

Several types of correlation function $R\left(x_{i}, x_{j}\right)$ can be used, and, in this study, the Gaussian function can be formulated as

$$
R\left(x_{i}, x_{j}\right)=\operatorname{EXP}\left(-\sum_{k=1}^{n_{\mathrm{dv}}} \theta_{k}\left|x_{k}^{i}-x_{k}^{j}\right|^{2}\right),
$$

where $n_{\mathrm{dv}}$ is the dimension of random input variables, $x_{k}^{i}$ and $x_{k}^{j}$ denote the $k$ th component of training samples $x_{i}$ and $x_{j}$, respectively, and $\theta_{k}$ is the correlation parameter to ensure that the metamodel is flexible enough to approximate the true function, which is usually obtained by an optimization process.

A set of $n_{s}$ experimental samples is denoted by $\mathbf{x}=$ $\left[\mathbf{x}_{1}, \mathbf{x}_{2}, \ldots, \mathbf{x}_{n_{s}}\right]^{T}$, where $\mathbf{x}_{i}$ is the $i$ th training point, and responses $\mathbf{Y}=\left[y_{1}, y_{2}, \ldots, y_{n_{\mathrm{s}}}\right]^{T}$ is the corresponding response to $\mathbf{x}$. The predicted value $\mu_{G}(\mathbf{x})$ and predicted variance $\sigma_{G}^{2}(\mathbf{x})$ of the objective function $G(\mathbf{x})$ at an unknown point $\mathbf{x}$ are

$$
\mu_{G}(\mathbf{x})=f^{T} \widehat{\beta}+r^{T}(\mathbf{x}) R^{-1}(Y-F \widehat{\beta}) .
$$

In (17), $R$ denotes a $m \times m$ correlation matrix by $R_{i, j}=$ $R\left(x_{i}, x_{j}\right) ; r^{T}(\mathbf{x})=\left[R\left(\mathbf{x}, \mathbf{x}_{1}\right), R\left(\mathbf{x}, \mathbf{x}_{2}\right), \ldots, R\left(\mathbf{x}, \mathbf{x}_{n_{\mathrm{s}}}\right)\right]^{T}$ is the correlative relations between unknown point $\mathbf{x}$ and sample points $\mathbf{x}=\left[\mathbf{x}_{1}, \mathbf{x}_{2}, \ldots, \mathbf{x}_{n_{s}}\right]^{T}$; the unknown parameters $\widehat{\beta}$ can be expressed as

$$
\widehat{\beta}=\left(F^{T} R^{-1} F\right)^{-1} F^{T} R^{-1} Y .
$$

The Kriging variance $\sigma_{G}^{2}(\mathbf{x})$ is defined as the minimum square error between true response $G(\mathbf{x})$ and estimated value $\widehat{G}(\mathbf{x})$ and is expressed as follows:

$$
\sigma_{G}^{2}(\mathbf{x})=\sigma_{z}^{2}\left(1+u^{T}\left(F^{T} R^{-1} F\right)^{-1} u-r^{T}(\mathbf{x}) R^{-1} r(\mathbf{x})\right),
$$

where $u=F^{T} R^{-1} r(\mathbf{x})-f$; the unknown parameters $\sigma_{z}^{2}$ can be expressed as

$$
\sigma_{z}^{2}=\frac{(Y-1 \widehat{\beta})^{T} R^{-1}(Y-1 \widehat{\beta})}{n_{s}} .
$$

However, at a given unknown point $\mathbf{X}$, the predicted value $\mu_{G}(\mathbf{X})$ is not the true value of $G(\mathbf{X})$ and there exist some uncertainties. $\sigma_{G}^{2}(\mathbf{x})$ demonstrates uncertainty of the predictor value; it also provides an important index to adjudge the fitting accuracy and enables quantifying the uncertainty of predictions with an easy approach. 
3.2. The ALK Method and the Solution for the Parametric Sensitivity of IM $\delta_{X_{i}}^{N P}$. The ALK method has been applied to different fields in engineering, like efficient global optimization (EGO) [22], probabilistic analysis (PRA) [24-26], and reliability-based design optimization [28]. In the ALK method, the learning function plays an important role in constructing the active learning Kriging model [26]. A new Kriging model is updated by adding a new training point to the design of experiment (DOE) in subsequent iterations by active learning until the Kriging model satisfies necessary accuracy. According to the indicator function of this failure domain in (5), we only need to focus on the sign of the performance function in the reliability analysis.

For the uncertainty of predicted value $\mu_{G}(\mathbf{X})$, there may exist some risk that the value of $G(\mathbf{X})$ is positive $(G(\mathbf{X})>0)$ even if predicted value is negative $\left(\mu_{G}(\mathbf{X})<0\right)$. So, the points owing a high potential risk to cross the predicted separator $G(\mathbf{X})=0$ have to be added to the DOE and evaluated by the real performance function. These potentially "dangerous" points determining the precision of the failure probability are in the region: close to the limit state, have high Kriging variance or both. To identify them, a new learning function named the expected risk function (ERF) is proposed in our recent work [29].

First, if $\mu_{G}(\mathbf{X})<0$, we define an indicator to measure such potential risk as

$$
R(\mathbf{X})=\max [(G(\mathbf{X})-0), 0] .
$$

As shown in Figure 2(a), $R(\mathbf{X})$ measures the shift of value of $G(\mathbf{X})$ to be positive and the larger the value of $R(\mathbf{X})$ is, the more risky the sign of $G(\mathbf{X})$ is to be changed from negative to positive.

Covering the range $(G(\mathbf{X})>0)$ to calculate the mean of $R(\mathbf{X})$, the ERF for the case $\mu_{G}(\mathbf{X})<0$ is obtained as follows:

$$
\begin{aligned}
E(R(\mathbf{X})) & =E(\max [(G(\mathbf{X})-0), 0]) \\
& =\int_{0}^{+\infty} G \phi\left(\frac{G-\mu_{G}}{\sigma_{G}}\right) d G \\
& =\sigma_{G} \phi\left(\frac{\mu_{G}}{\sigma_{G}}\right)+\mu_{G} \Phi\left(\frac{\mu_{G}}{\sigma_{G}}\right),
\end{aligned}
$$

where $\mu_{G}$ and $\sigma_{G}$ are the predicted value and variance in (17) and (19) and $\Phi(\cdot)$ and $\phi(\cdot)$ are the cumulative distribution function $(\mathrm{CDF})$ and PDF of the standard normal distribution.

If $\mu_{G}(\mathbf{X})>0$, as shown in Figure 2(b), we define an indicator to measure such potential risk as

$$
R(\mathbf{X})=\max [(0-G(\mathbf{X})), 0] .
$$

And the ERF for the case $\mu_{G}(\mathbf{X})>0$ is obtained as follows:

$$
\begin{aligned}
E(R(\mathbf{X})) & =E(\max [(0-G(\mathbf{X})), 0]) \\
& =\int_{0}^{+\infty} G \phi\left(\frac{G+\mu_{G}}{\sigma_{G}}\right) d G \\
& =\sigma_{G} \phi\left(\frac{\mu_{G}}{\sigma_{G}}\right)-\mu_{G} \Phi\left(\frac{\mu_{G}}{\sigma_{G}}\right) .
\end{aligned}
$$

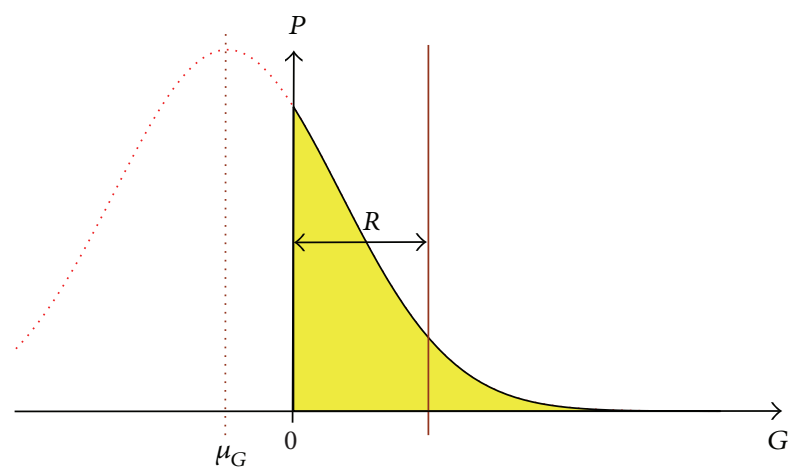

(a)

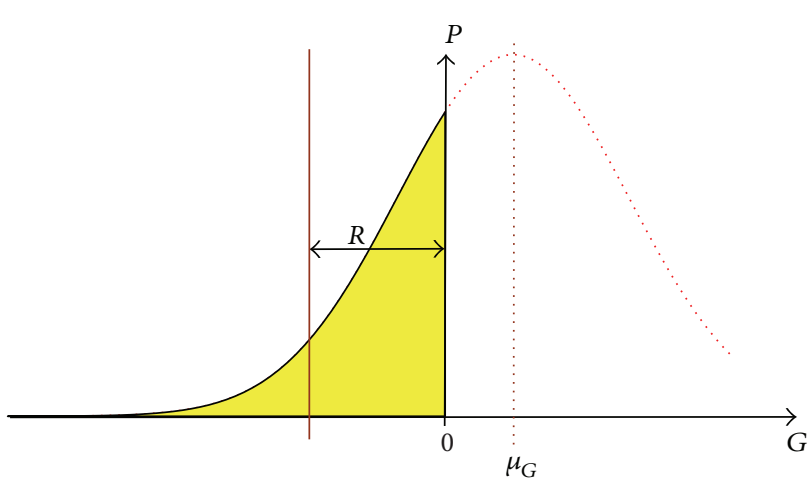

(b)

FIGURE 2: Risk of the sign of $G(\mathbf{X})$ to be wrongly predicted in a Kriging model: (a) the sign of $G(\mathbf{X})$ is negative; (b) the sign of $G(\mathbf{X})$ is positive.

The ERF is employed to measure the potential possibility that the sign of the limit state function in a point $\mathbf{X}$ is changed from positive to negative (or negative to positive) in the Kriging model. The point maximizing ERF should be added to the initial set of training points in DOE.

The ALK-based solution for the parametric sensitivity can be simply divided into five steps and provided as follows.

Step 1. For a model $y=g(\mathbf{x})$, generate $N$ samples $\mathbf{x}_{t}=$ $\left(x_{1 t}, x_{2 t} \cdots x_{n t}\right)(t=1,2 \cdots N)$ of the input variables by PDF $f_{\mathbf{X}}(\mathbf{x})$ using Sobol's low-discrepancy samples. One can refer to Sobol [15] for more details. A $n \times N$ dimension matrix $\mathbf{X}$ is formed and shown as

$$
\mathbf{X}=\left(\mathbf{x}_{1}, \mathbf{x}_{2}, \ldots, \mathbf{x}_{N}\right)^{T}=\left[\begin{array}{ccc}
x_{1,1} & \cdots & x_{n, 1} \\
\vdots & \ddots & \vdots \\
x_{1, N} & \cdots & x_{n, N}
\end{array}\right]
$$

where the $i$ th column of matrix $\mathbf{X}$ represents the generated random realizations for input variable $x_{i}$.

Step 2. Construct an active learning Kriging model as follows.

(a) The samples $\mathbf{x}_{t}$ in Step 1 are set as candidate points. Randomly choose some training points in the samples $\mathbf{x}_{t}$ and evaluate the corresponding performance 
function. In the initial step, only a dozen of points in the DOE are enough according to our experience.

(b) Construct the Kriging model, compute the ERF of candidate points by (22) and (24), and judge whether it is smaller than the given tolerance. If so, the active learning process can stop and turn to Step 3 directly. If the stopping condition is not satisfied, it should go to Step 2(c) to add new training point.

(c) Add the point with max value of ERF to the DOE and loop back to Step 2(b).

Step 3. Based on the active learning Kriging model, the corresponding $N$ values of Kriging predictions $\mathbf{y}_{t}=$ $\mu_{G}\left(\mathbf{x}_{t}\right)(t=1,2 \cdots N)$ are obtained. Compute the values $\mathbf{I}_{F}^{t}(t=1,2 \cdots N)$ of the indicator function of this failure domain and $P_{f_{Y}}=\sum_{t=1}^{N} I_{F}^{t} / N$. Using the same active learning Kriging model, $\partial P_{f_{Y}} / \partial \theta_{X_{i}}^{j}$ and $\partial P_{f_{Y}} / \partial \theta_{X_{k}}^{j}$ can be computed combining the reliability sensitivity analysis method by (10).

Step 4. By fixing the $(i, t)$ th component $\left(x_{i, t}\right)$ of $\mathbf{X}$, generate $M$ samples $\mathbf{v}_{x_{i t}}^{j}=\left(x_{1 j}, \ldots, x_{i-1, j}, x_{i t}, x_{i+1, j} \cdots x_{n j}\right)(j=$ $1,2 \cdots M)$ according to the conditional PDF $f_{\mathbf{X}}\left(\mathbf{x} \mid x_{i t}\right)$, and the corresponding $M$ values of Kriging predictions $\mathbf{y}_{x_{i, t}}^{j}=$ $\mu_{G}\left(\mathbf{v}_{x_{i, t}}^{j}\right)(j=1,2 \cdots M)$ can be obtained based on the same active learning Kriging model. With these $M$ samples $\mathbf{y}_{x_{i t}}^{j}(j=$ $1,2 \cdots M)$, the $P_{f_{Y} \mid x_{i t}}$ of conditional on $x_{i t}$ can be obtained as

$$
P_{f_{Y} \mid x_{i t}}=\frac{\sum_{j=1}^{M} I_{F \mid x_{i t}}^{j}}{M}
$$

At the same time, $P_{f_{Y \mid X_{i}}} / \partial \theta_{X_{k}}^{j}$ can be computed combining the reliability sensitivity analysis method by (11).

Step 5. The IM $\delta_{X_{i}}^{P}$ can be calculated according to the definition of (2); $P_{f_{Y}}, P_{f_{Y} \mid x_{i t}}, \partial P_{f_{Y}} / \partial \theta_{X_{i}}^{j}, \partial P_{f_{Y}} / \partial \theta_{X_{k}}^{j}$, and $P_{f_{Y \mid X_{i}}} / \partial \theta_{X_{k}}^{j}$ have been computed in Steps 3 and 4. Substituting them into (8) or (9), the parametric sensitivity of IM $\delta_{X_{i}}^{N P}$ can be computed.

It can be seen that a large number of samples must be taken for providing precise estimates when calculating IM $\delta^{P}$ and its parametric sensitivity with Monte Carlo method. In the second step of ALK procedure, one only needs to compute the limit state function values using the metamodel instead of original model; thus, this procedure can further improve the efficiency without loss of precision, especially for the finite element model (FEM).

In the next section, we introduce two numerical examples and two engineering examples for demonstrating the efficiency and precision of the calculation procedure and illustrating the engineering significance of the IM $\delta^{P}$ and its parametric sensitivity.
TABLE 1: Computational results of the importance measure $\delta_{x_{i}}^{P}$ in Example 1.

\begin{tabular}{lccc}
\hline Method & \multicolumn{2}{c}{ Importance measure $\delta_{x_{i}}^{P}$} & $\begin{array}{c}\text { The numbers of } \\
\text { function evaluations }\end{array}$ \\
\hline MC & $x_{1}$ & $x_{2}$ & $2 \times 10^{4} \times 10^{4}$ \\
ALK & 0.00492 & 0.00537 & $12+36$ \\
Error & 0.00476 & 0.00548 & \\
\hline
\end{tabular}

\section{Examples}

\subsection{Numerical Examples}

Example 1. The mathematical problem in Example 1 is modified from [25], which behaves nonlinearly around the limit state function. The nonlinear performance function is given as

$$
g\left(x_{1}, x_{2}\right)=\sin \left(\frac{5 x_{1}}{2}\right)-\frac{\left(x_{1}^{2}+4\right)\left(x_{2}-1\right)}{20}+2
$$

where the basic variables $x_{1}$ and $x_{2}$ are independent and follow normal distributions $x_{1} \sim N(1.5,1)$ and $x_{2} \sim$ $N(2.5,1)$, respectively.

The estimates of the importance measure on the failure probability $\delta_{X_{i}}^{P}$ computed by the MCS and ALK procedures and the error in each estimate are reported in Table 1 . The numbers in the last column are the evaluation numbers of the performance function when calculating $\delta_{X_{i}}^{P}$. The results of their parametric sensitivities are computed using the same active learning Kriging model and listed in Table 2.

As revealed by Tables 1 and 2, the results computed by the ALK procedure are precise enough compared with those computed by the MCS procedure. The results of MCS procedure can be seen as the accurate results. Compared with the tremendously large computational cost $\left(2 \times 10^{4} \times 10^{4}\right.$ samples) of Monte Carlo method, the ALK method only begins with 12 training points, while 36 points whose sign of response has the largest potential risk to be wrongly predicted are added into the initial DOE. Additionally, Figure 3 gives iteration history of the true values of the 36 training points for Example 1 by the ALK method. The sign of the candidate points (the samples in MCS procedure) is estimated using the ALK model. Compared with the true sign of the candidate points using originality performance function, there are only 8 candidate points whose signs of responses are wrongly predicted. This can be linked to Figure 4 in which it shows the sign of the response at each Monte Carlo sampling point. It is seen that most of the added points are located in the vicinity of the limit state function. Consequently, this method certifies a correct approximation of the response using a minimum number of calls to the performance function.

From the results in Table 1, it is noted that there is no difference between the rankings of the basic variables on failure probability by the MCS and ALK procedures, namely $x_{2}>x_{1}$, which illustrates that the ALK method is meaningful and reasonable. Therefore, $x_{2}$ should be paid more attention 
TABLE 2: The parametric sensitivities of the importance measure $\delta_{x_{i}}^{P}$ in Example 1 .

\begin{tabular}{|c|c|c|c|c|c|}
\hline Parametric sensitivity of IM & Method & $\mu_{x_{1}}$ & $\sigma_{x_{1}}$ & $\mu_{x_{2}}$ & $\sigma_{x_{2}}$ \\
\hline$\partial \delta_{x_{1}}^{P}$ & $\mathrm{MC}$ & 0.00821 & 0.0179 & 0.00940 & 0.00610 \\
\hline$\overline{\partial \theta_{x_{i}}}$ & ALK & 0.00858 & 0.0183 & 0.00947 & 0.00624 \\
\hline$\partial \delta_{x_{2}}^{P}$ & $\mathrm{MC}$ & 0.00741 & -0.00301 & $4.594 e^{-4}$ & $-3.576 e^{-4}$ \\
\hline$\overline{\partial \theta_{x_{i}}}$ & ALK & 0.00752 & -0.00293 & $4.648 e^{-4}$ & $-3.470 e^{-4}$ \\
\hline
\end{tabular}

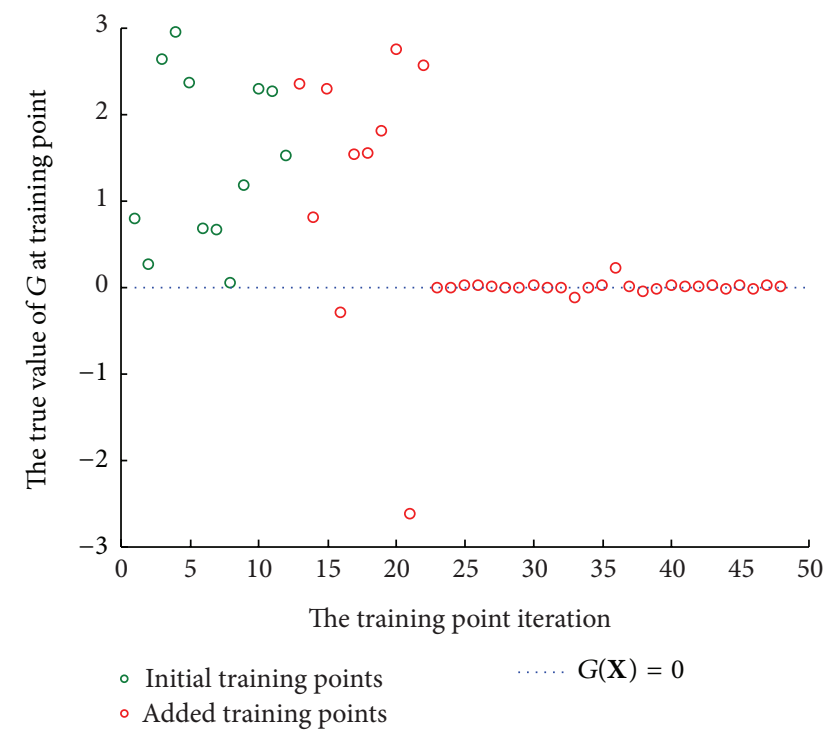

FIGURE 3: Iteration history of the true value of added training points for Example 1 by ALK.

to in the reliability analysis on failure probability. Results shown in Table 2 point out the influences of varying some variables' distribution parameters to the IMs on the failure probability. The uncertainty of IMs $\delta_{x_{i}}^{P}$ on failure probability can be improved through modifying or controlling the distribution parameters of the variables indirectly.

Example 2 (Ishigami Function). Ishigami function [30] is a commonly used example in importance measure analysis [6, 20]. It can be written as follows:

$$
g=\sin x_{1}+a \sin ^{2} x_{2}+b x_{3}^{4} \sin x_{1},
$$

where the basic variables $x_{1}, x_{2}$, and $x_{3}$ are independent and uniformly distributed in $\left[x_{i}^{L}, x_{i}^{U}\right](i=1,2,3), x_{i}^{L}=-\pi$, and $x_{i}^{U}=\pi$. The values of constants are set as $a=5$ and $b=0.1$. The estimates of the importance measure on failure probability $\delta_{X_{i}}^{P}$ and the results of their parametric sensitivities are listed in Tables 3 and 4, respectively.

As revealed by Tables 3 and 4 , the computational cost of Monte Carlo method $\left(3 \times 10^{8}\right.$ samples) is tremendously large, which illustrates that our model is computationally challenging. The ALK method begins with 12 training points, while 44 points are added into the initial DOE to satisfy the accuracy. We plot the tendency of the true values of
TABLE 3: Computational results of the importance measure $\delta_{x_{i}}^{P}$ in Ishigami function.

\begin{tabular}{lccc}
\hline \multirow{2}{*}{ Method } & \multicolumn{3}{c}{ Importance measure $\delta_{x_{i}}^{P}$} \\
& $x_{1}$ & $x_{2}$ & $x_{3}$ \\
\hline MC $\left(6 \times 10^{4} \times 10^{3}\right)$ & 0.0474 & 0.0263 & 0.0107 \\
ALK $\left(6 \times 10^{4} \times 10^{3}\right)$ & 0.0475 & 0.0261 & 0.0110 \\
Error & 0.0053 & 0.0076 & 0.028 \\
\hline
\end{tabular}

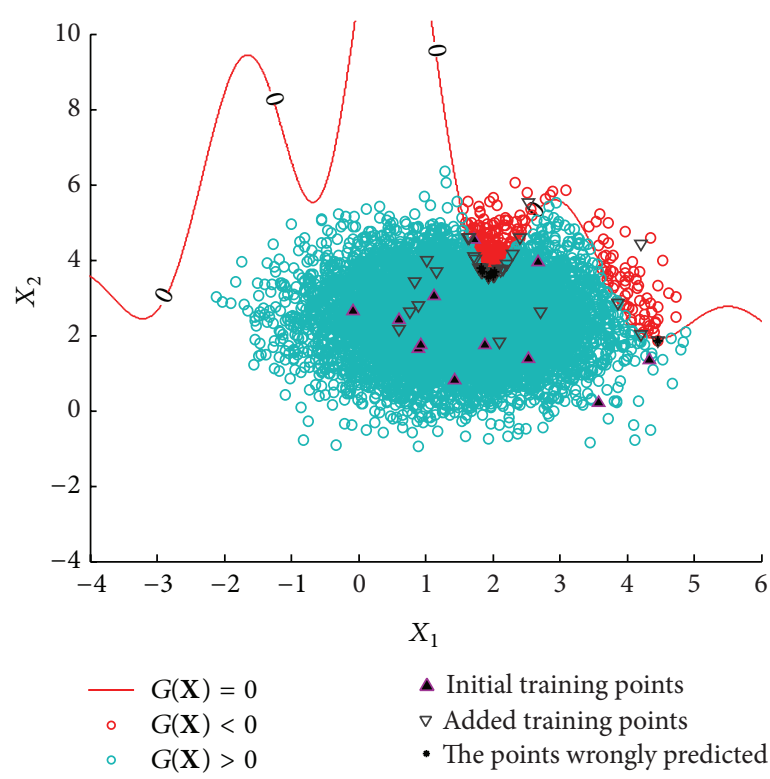

FIgURE 4: Sign of the response at each sample predicted for Example 1 by ALK.

the training points for Ishigami function computed by ALK method in Figure 5. This validates the high efficiency of the ALK method. The importance ranking of the basic variables on failure probability obtained by $\delta_{X_{i}}^{P}$ is $x_{1}, x_{2}$, and $x_{3}$. Therefore, $x_{1}$ should be paid more attention to in the reliability analysis on failure probability. Results shown in Table 4 point out the influences of varying some variables' distribution parameters to the IMs on the failure probability.

From Examples 1 and 2, it can be found that the importance of a variable to failure probability is not just affected by the distribution parameters of this variable but affected by those of the rest of input variables, and sometimes the latter may even play a more important role than the former. Surely, the engineering examples in Sections 4.2 and 4.3 are also following the fact. Accordingly, it is necessary to 
TABLE 4: The parametric sensitivities of the importance measure $\delta_{x_{i}}^{P}$ in Ishigami function.

\begin{tabular}{|c|c|c|c|c|c|c|c|}
\hline Parametric sensitivity of IM & Method & $x_{1}^{L}$ & $x_{1}^{U}$ & $x_{2}^{L}$ & $x_{2}^{U}$ & $x_{3}^{L}$ & $x_{3}^{U}$ \\
\hline$\partial \delta_{x_{1}}^{P}$ & $\mathrm{MC}$ & $-7.826 e^{-3}$ & $7.826 e^{-3}$ & $1.513 e^{-2}$ & $-1.513 e^{-2}$ & $1.513 e^{-2}$ & $-1.513 e^{-2}$ \\
\hline$\overline{\partial \theta_{x_{i}}}$ & ALK & $-7.752 e^{-3}$ & $7.752 e^{-3}$ & $1.537 e^{-2}$ & $-1.537 e^{-2}$ & $1.537 e^{-2}$ & $-1.537 e^{-2}$ \\
\hline$\partial \delta_{x_{2}}^{P}$ & MC & $8.422 e^{-3}$ & $-8.422 e^{-3}$ & $-4.402 e^{-3}$ & $4.402 e^{-3}$ & $8.422 e^{-3}$ & $-8.422 e^{-3}$ \\
\hline$\overline{\partial \theta_{x_{i}}}$ & ALK & $8.418 e^{-3}$ & $-8.418 e^{-3}$ & $-4.398 e^{-3}$ & $4.398 e^{-3}$ & $8.418 e^{-3}$ & $-8.418 e^{-3}$ \\
\hline$\partial \delta_{x_{3}}^{P}$ & $\mathrm{MC}$ & $3.425 e^{-3}$ & $-3.425 e^{-3}$ & $3.425 e^{-3}$ & $-3.425 e^{-3}$ & $-1.896 e^{-3}$ & $1.896 e^{-3}$ \\
\hline$\overline{\partial \theta_{x_{i}}}$ & ALK & $3.479 e^{-3}$ & $-3.479 e^{-3}$ & $3.480 e^{-3}$ & $-3.479 e^{-3}$ & $-1.914 e^{-3}$ & $-1.914 e^{-3}$ \\
\hline
\end{tabular}

TABLE 5: Statistical properties of random variables for roof truss.

\begin{tabular}{lcccccc}
\hline Random variable & $q(\mathrm{~N} / \mathrm{m})$ & $l(\mathrm{~m})$ & $A_{S}\left(\mathrm{~m}^{2}\right)$ & $A_{C}\left(\mathrm{~m}^{2}\right)$ & $E_{S}(\mathrm{~Pa})$ & $E_{C}(\mathrm{~Pa})$ \\
\hline Mean $\mu_{x}$ & 20000 & 12 & $9.82 \times 10^{-4}$ & 0.04 & $1 \times 10^{11}$ & $1.2 \times 10^{10}$ \\
\hline Coefficient of variation $\mathrm{Cov}_{x}$ & 0.07 & 0.01 & 0.06 & 0.12 & 0.06 & 0.06 \\
\hline
\end{tabular}

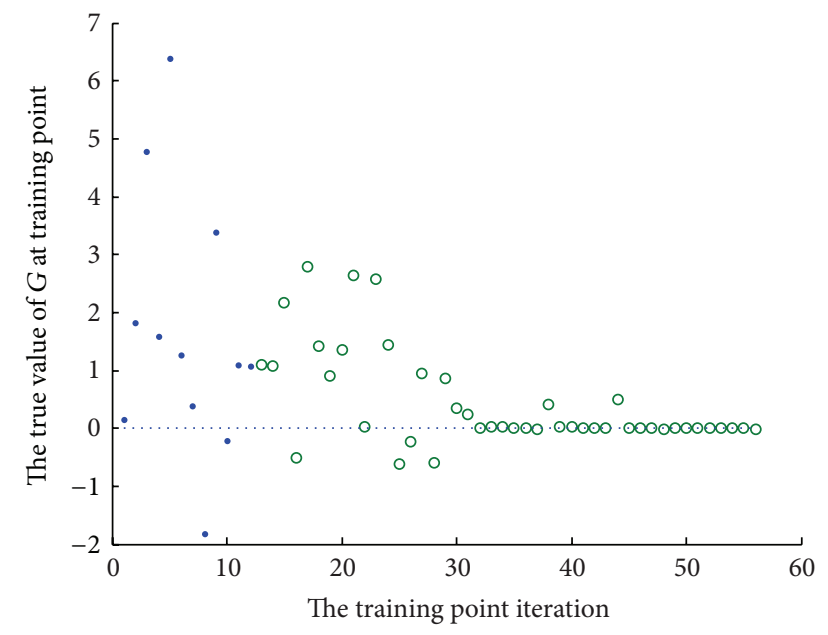

- Initial training point

- Added training point

FIGURE 5: Iteration history of the true value of added training points for Ishigami function by ALK.

find out all the factors that may affect the importance measures according to the failure probability, and parametric sensitivities on IMs provide a way to solve this problem.

Except for that, Table 4 also shows an important property as all the variables of the model are uniform variables; namely, the numerical values of two parametric sensitivities of the IM are equal, and their signs are opposite. This property result of the parametric sensitivity of the IM is decided by (8) and (9). For the uniform variable, the signs of $\left(1 / f_{\mathbf{X}}(\mathbf{x})\right)\left(\partial f_{\mathbf{X}}(\mathbf{x}) / \partial \theta_{X_{i}}\right)$ are opposite.

4.2. Roof Truss. In order to test the applicability of the proposed method for problems with more random variables and expressed in a more engineering way, the roof truss is selected as example. The truss is simply illustrated as in Figure 6. The top chord and the compression bars of the truss are reinforced by concrete and the bottom chord and the tension bars are all made of steel. Assume the truss bears uniformly distributed load $q$, which can be transformed into nodal load $P=q l / 4$. The perpendicular deflection of truss peak node $C \Delta_{C}$ can be calculated using the knowledge of structural mechanics and

$$
\Delta_{C}=\frac{q l^{2}}{2}\left(\frac{3.81}{A_{C} E_{C}}+\frac{1.13}{A_{S} E_{S}}\right)
$$

where $A_{C}$ and $A_{S}$ are the cross-sectional areas of the reinforced concrete and steel bars, respectively, $E_{C}$ and $E_{S}$ are the corresponding elastic moduli of reinforced concrete and steel, and $l$ is the length of the truss as Figure 6 shows. The distribution parameters of the independent normal random basic variables are given in Table 5 .

Considering the safety of the truss, the perpendicular deflection $\Delta_{C}$ should satisfy the constraint $\Delta_{C} \leq 0.03 \mathrm{~m}$. Hence, the structural performance function can be given as follows:

$$
g(x)=0.03-\Delta_{C}=0.03-\frac{q l^{2}}{2}\left(\frac{3.81}{A_{C} E_{C}}+\frac{1.13}{A_{S} E_{S}}\right) .
$$

For this highly nonlinear example, the computational results of the importance measure on failure probability measures by ALK and MCS are listed in Tables 6 and 7 . In addition, the IMs on the failure probability by the state dependent parameter (SDP) method in literature [20] are also listed for comparison in Table 6. The MCS procedure takes $6 \times 10^{7}$ samples. The SDP method needs only 1024 model runs for calculating the importance measures. However, the Kriging method needs to call the performance function only 72 times. The ALK method begins with 12 training points, and 60 points are added into the initial DOE using ALK. The tendency of the true values of the training points for roof truss by ALK method is plotted in Figure 7. Compared with the SDP method, the proposed method has obtained a more accurate result and lower computational cost.

Additionally, it can be seen from Table 6 that the effects of the basic variable of $q$ on the failure probability are notable and the influences of basic variables sectional area $A_{C}$ and $A_{S}$ 
TABLE 6: Computational results of the importance measure $\delta_{x_{i}}^{P}$ for roof truss.

\begin{tabular}{|c|c|c|c|c|c|c|}
\hline \multirow{2}{*}{ Method } & \multicolumn{6}{|c|}{ Global reliability sensitivity indices $\delta_{x_{i}}^{P}$} \\
\hline & $q$ & $l$ & $A_{C}$ & $A_{S}$ & $E_{C}$ & $E_{S}$ \\
\hline $\mathrm{MC}\left(6 \times 10^{4} \times 10^{3}\right)$ & 0.05714 & 0.00483 & 0.02431 & 0.01148 & 0.00664 & 0.01329 \\
\hline ALK (69) & 0.05684 & 0.00471 & 0.02425 & 0.01151 & 0.00665 & 0.01328 \\
\hline SDP (1024) & 0.05044 & 0.00436 & 0.02749 & 0.01334 & 0.00691 & 0.01231 \\
\hline
\end{tabular}

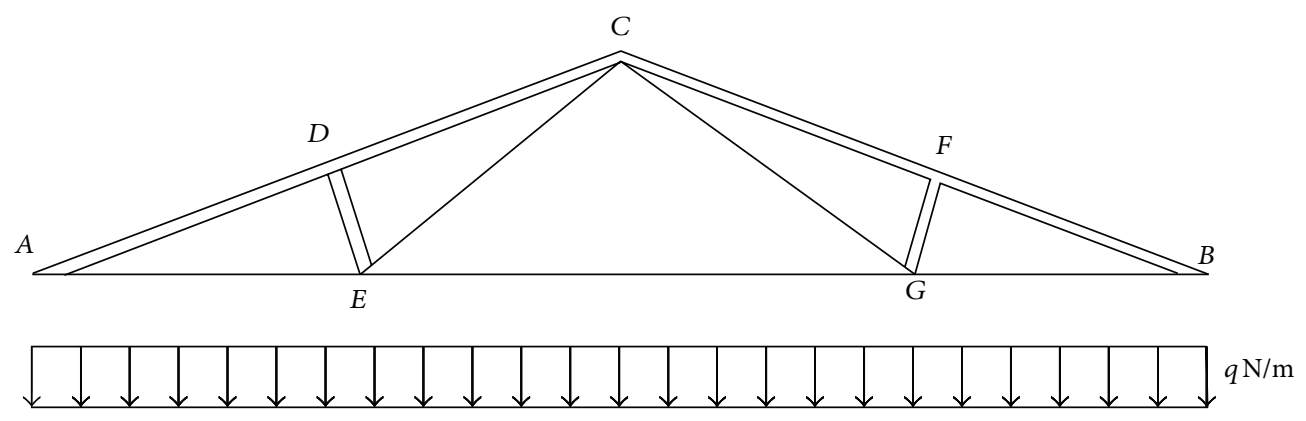

(a)

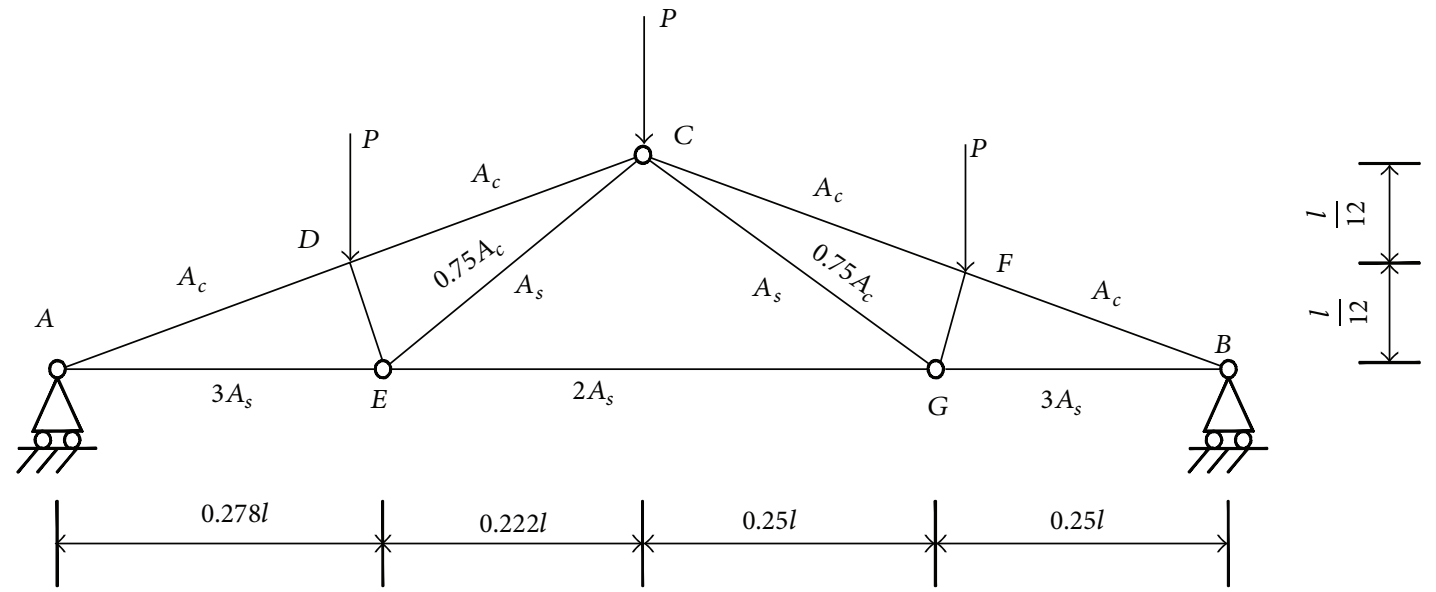

(b)

FIGURE 6: Schematic diagram of a roof truss.

on the failure probability are relatively smaller, whereas elastic moduli $E_{S}$ and $E_{C}$ and the length $l$ are the least influential ones which can attract less attention. In engineering, parameters of the load $q$ and the length $l$ are more easily controlled or modified than other inputs, so the paper especially pays attention to their parametric sensitivities of $\operatorname{IM} \delta_{X_{i}}^{P}$.

Due to space limitation, Table 7 lists the parametric sensitivities of basic variables IM on the failure probability. Taking IM $\delta_{q}^{P}$ for example, the distribution parameters of the load $q$ are relatively higher than those of the length $l$. Thus, it can be seen that changing those parameters with high parametric sensitivity has more influences on the IM of the corresponding variables than other parameters. The parametric sensitivity of $\delta_{X_{i}}^{P}$ is valuable in the reliability engineering because it can provide indirect information for reliability design and reliability-based optimization.
4.3. A Planar 10-Bar Structure. A planar 10-bar structure shown in Figure 8 is investigated. The horizontal bars have the same length $L=1 \mathrm{~m}$. The diagonal bars and the vertical members have the same length $\sqrt{2} L$. The cross-sectional area of 10 bars is denoted by $A=0.001 \mathrm{~m}^{2}$. The elastic modulus of all bars is $E=100 \mathrm{GPa} . P_{1}=80 \mathrm{KN}$ and $P_{2}=10 \mathrm{KN}$ are the external loads subjected to joints 4 and 2 , respectively. Joint 2 is also subjected to a horizontal load $P_{3}=10 \mathrm{KN}$. The input variables are all normally distributed, and their coefficient of variance is 0.05 . We assume the displacement of node 3 in vertical direction not exceeding $3 \mathrm{~mm}$ as the constrain condition. The limit state function can be constructed, $g=3 \mathrm{~mm}-\left|Y_{2}\right|$, where $Y_{2}$ is an implicit function of the basic random variables. As shown in Figure 9, the finite element model can be obtained in Ansys 11.0. The results of the importance measures $\delta^{P}$ of inputs are listed in Table 8 . To identify the influential distribution parameters, 
TABLE 7: The parametric sensitivities of the importance measure $\delta_{x_{i}}^{P}$ for roof truss.

\begin{tabular}{|c|c|c|c|c|c|}
\hline \multirow{2}{*}{ Parametric sensitivity of IM } & \multirow{2}{*}{ Method } & \multicolumn{2}{|c|}{$l$} & \multicolumn{2}{|c|}{$q$} \\
\hline & & $\mu_{l}$ & $\sigma_{l}$ & $\mu_{q}$ & $\sigma_{q}$ \\
\hline$\partial \delta_{q}^{P}$ & $\mathrm{MC}$ & $2.361 e^{-6}$ & $-1.369 e^{-6}$ & $2.241 e^{-5}$ & $5.221 e^{-5}$ \\
\hline$\frac{q}{\partial \theta_{x_{i}}}$ & ALK & $2.384 e^{-6}$ & $-1.378 e^{-6}$ & $2.256 e^{-5}$ & $5.240 e^{-5}$ \\
\hline$\partial \delta_{A_{C}}^{P}$ & $\mathrm{MC}$ & -0.432 & -0.285 & -4.947 & -4.053 \\
\hline$\overline{\partial \theta_{x_{i}}}$ & ALK & -0.428 & -0.271 & -4.956 & -4.057 \\
\hline$\partial \delta_{A_{S}}^{P}$ & $\mathrm{MC}$ & -28.546 & -7.802 & $-3.235 e^{2}$ & $-1.324 e^{2}$ \\
\hline$\frac{A_{S}}{\partial \theta_{x_{i}}}$ & ALK & -27.611 & -8.096 & $-3.312 e^{2}$ & $-1.387 e^{2}$ \\
\hline
\end{tabular}

TABLE 8: Computational results of the importance measure $\delta_{x_{i}}^{P}$ in 10-bar structure.

\begin{tabular}{lccccc}
\hline \multirow{2}{*}{ Method } & \multicolumn{5}{c}{ Global reliability sensitivity indices $\delta_{x_{i}}^{P}$} \\
& $A$ & $E$ & $l$ & $P_{1}$ & $P_{2}$ \\
\hline MC $\left(6 \times 10^{4} \times 10^{3}\right)$ & 0.0449 & 0.0382 & 0.0401 & 0.0242 & 0.00282 \\
ALK $(69)$ & 0.0443 & 0.0371 & 0.0396 & 0.0245 & 0.00265 \\
\hline
\end{tabular}

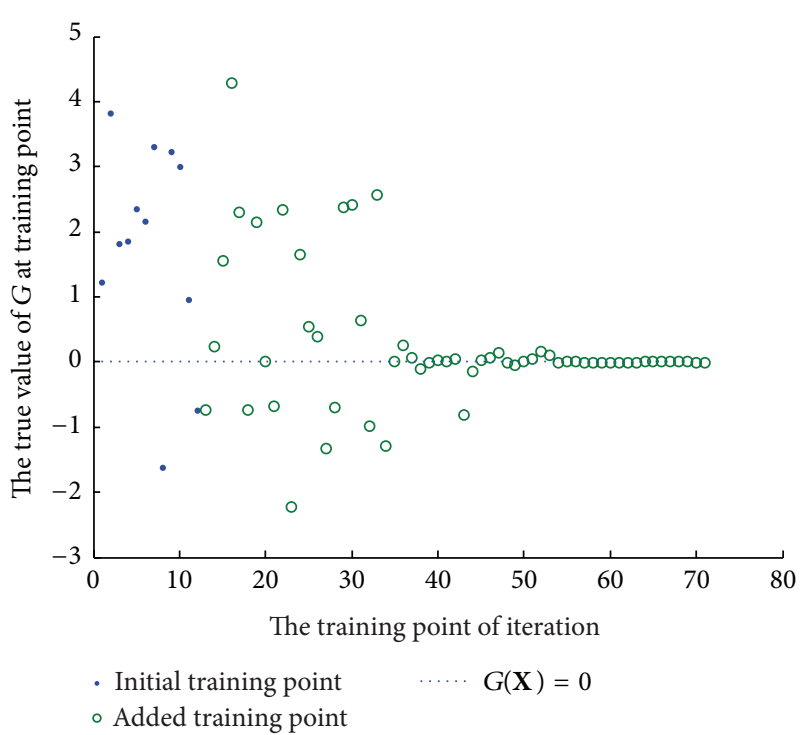

FIGURE 7: Iteration history of the true value of added training points for a roof truss by ALK.

the computational results of parametric sensitivity are shown in Table 9.

From Tables 8 and 9, it can be seen that the computational results of Monte Carlo method and ALK method are in good agreement. The MCS procedure takes $6 \times 10^{7}$ samples and its results can be seen as the accurate results, but the computational cost of Monte Carlo method is tremendously large especially for FEM. The ALK method only needs to call the finite element model 93 times to satisfy the accuracy. The efficient Kriging method begins to get convergence at 12 training points, and 81 points are added into the initial DOE using ALK. The true values of the added training points for roof truss by the ALK method are shown in Figure 10. The ALK method can also improve the computational efficiency impressively and ensure acceptable accuracy for the implicit finite element model.

As revealed in Table 8, the ranking of IM not to exceed as $3 \mathrm{~mm}$ the constrain condition $\delta^{P}$ is as follows: the basic variables of $A, l$, and $E$ on the failure probability are notable; the influences of basic variable $P_{1}$ on the failure probability are less; and the influences of basic variables $P_{2}$ and $P_{3}$ on the failure probability are very small, which are even near zeros. From Table 9, the distribution parameters $\mu_{A}, \sigma_{A}$, $\mu_{l}$, and $\sigma_{l}$ are the most influential ones on the IMs. Thus, it can be seen that changing those parameters with high parametric sensitivities has more influences on the IM of the corresponding variable than other parameters. In the sight of this structural design, we need to pay more attention to the distribution parameters with high parametric sensitivity. In order to obtain the IM on failure probability results, especially for the high ranking IM, the important distribution parameters to them must be given precisely. To do this, it is necessary to collect the information and improve the understanding of the distribution parameters.

\section{Conclusions}

This paper investigates the influence of the distribution parameters on the IM on failure probability. It is noted that the IM of basic variable not only is influenced by its distribution parameters but also is influenced by other basic variables' distribution parameters. By further developing the presented moment-independent IM on failure probability, the parametric sensitivity of IM is first presented according to the derivative theory; thus, how the influential distribution parameters influence the influential IM can be made clear. Meanwhile, we can decrease the variability of the IM on failure probability by collecting the information and improving the understanding of those most influential parameters. The parametric sensitivity of $\delta_{X_{i}}^{P}$ is valuable in reliability engineering because it can provide direct 
TABLE 9: The parametric sensitivities of the importance measure $\delta_{x_{i}}^{P}$ in 10-bar structure.

\begin{tabular}{|c|c|c|c|c|c|c|c|}
\hline \multirow[t]{2}{*}{ Basic variable } & \multirow[t]{2}{*}{$\theta_{x_{i}}$} & \multicolumn{2}{|c|}{$\frac{\partial \delta_{A}^{P}}{\partial \theta_{x_{i}}}$} & \multicolumn{2}{|c|}{$\frac{\partial \delta_{E}^{P}}{\partial \theta_{x_{i}}}$} & \multicolumn{2}{|c|}{$\frac{\partial \delta_{l}^{P}}{\partial \theta_{x_{i}}}$} \\
\hline & & MC & ALK & $\mathrm{MC}$ & ALK & MC & ALK \\
\hline \multirow{2}{*}{$A$} & $\mu_{l}$ & -236.763 & -240.579 & 19.812 & 19.820 & 17.774 & 17.756 \\
\hline & $\sigma_{l}$ & 1235.778 & 1232.543 & -1.637 & -1.625 & -1.471 & -1.457 \\
\hline \multirow{2}{*}{$l$} & $\mu_{l}$ & 0.230 & 0.241 & 0.223 & 0.220 & $-1.759 e^{-2}$ & $-1.788 e^{-2}$ \\
\hline & $\sigma_{l}$ & -0.408 & -0.412 & -0.384 & -0.397 & $-1.956 e^{-2}$ & $-2.012 e^{-2}$ \\
\hline \multirow{2}{*}{ E } & $\mu_{l}$ & $-1.843 e^{-10}$ & $-2.316 e^{-10}$ & $1.477 e^{-11}$ & $1.546 e^{-11}$ & $-2.304 e^{-12}$ & $-2.277 e^{-12}$ \\
\hline & $\sigma_{l}$ & $-4.231 e^{-10}$ & $-6.186 e^{-10}$ & $-3.714 e^{-13}$ & $-4.827 e^{-13}$ & $-4.034 e^{-12}$ & $-5.259 e^{-12}$ \\
\hline \multirow{2}{*}{$F_{1}$} & $\mu_{l}$ & $2.186 e^{-5}$ & $2.492 e^{-5}$ & $2.127 e^{-6}$ & $2.111 e^{-6}$ & $2.620 e^{-6}$ & $2.581 e^{-6}$ \\
\hline & $\sigma_{l}$ & $-3.262 e^{-5}$ & $-3.641 e^{-5}$ & $-3.208 e^{-5}$ & $-3.496 e^{-5}$ & $-3.207 e^{-6}$ & $-3.834 e^{-6}$ \\
\hline \multirow{2}{*}{$F_{2}$} & $\mu_{l}$ & $6.210 e^{-6}$ & $7.434 e^{-6}$ & $5.812 e^{-6}$ & $5.792 e^{-6}$ & $7.420 e^{-6}$ & $6.834 e^{-6}$ \\
\hline & $\sigma_{l}$ & $-3.174 e^{-5}$ & $-3.412 e^{-5}$ & $-3.986 e^{-5}$ & $-3.796 e^{-5}$ & $-3.528 e^{-5}$ & $-3.419 e^{-5}$ \\
\hline \multirow{2}{*}{$F_{3}$} & $\mu_{l}$ & $-1.641 e^{-6}$ & $-1.624 e^{-6}$ & $-1.574 e^{-6}$ & $-1.657 e^{-6}$ & $-1.871 e^{-6}$ & $-1.862 e^{-6}$ \\
\hline & $\sigma_{l}$ & $-2.631 e^{-7}$ & $-2.942 e^{-7}$ & $-1.876 e^{-6}$ & $-1.748 e^{-6}$ & $2.576 e^{-7}$ & $2.124 e^{-7}$ \\
\hline
\end{tabular}

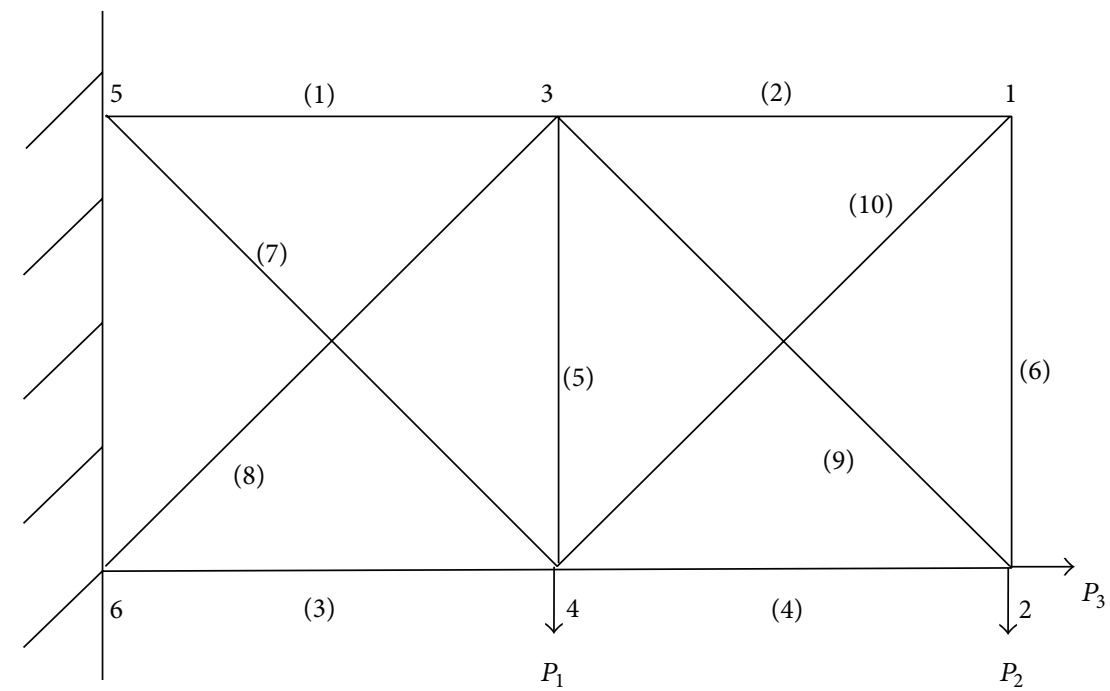

Figure 8: Planar 10-bar structure.

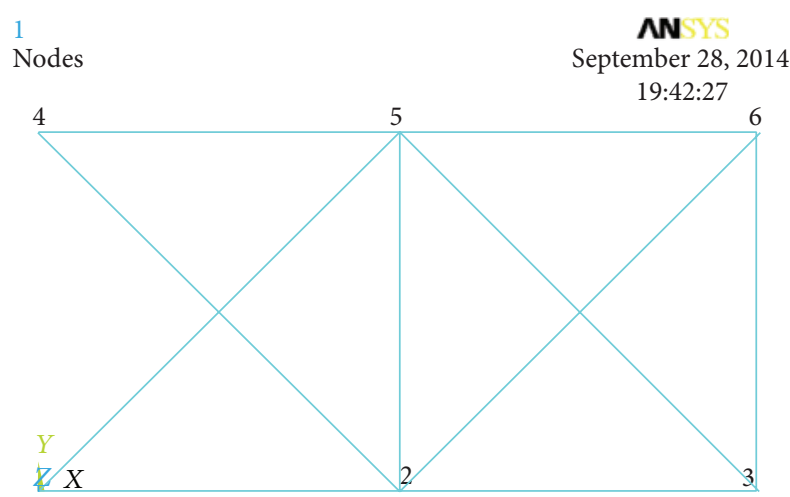

Figure 9: The finite element model of the planar 10-bar structure.

and useful information for reliability design and reliabilitybased optimization.
The computation of the IM on failure probability and its parametric sensitivity is often feasible by the MCS, but the computational cost of MCS method is tremendously large with small failure probability $\left(10^{-3}-10^{-4}\right.$ or smaller). For dealing with this problem, the ALK method is employed to calculate the IM and its parametric sensitivity. It can be seen by the numerical and engineering examples that the ALK method is more efficient than MC method. To ensure the computational accuracy, the large number of training points used in the traditional Kriging method is essential. Thanks to the existence of active learning process, the points which may greatly affect the metamodel's fitting accuracy can be precisely selected, which can make the Kriging metamodel more accurate and the additional computational cost is acceptable. It is noticed that a small quantity of points in the interesting region are added to construct the Kriging predictor model until the Kriging model satisfies necessary accuracy. The computational results of several examples demonstrate 


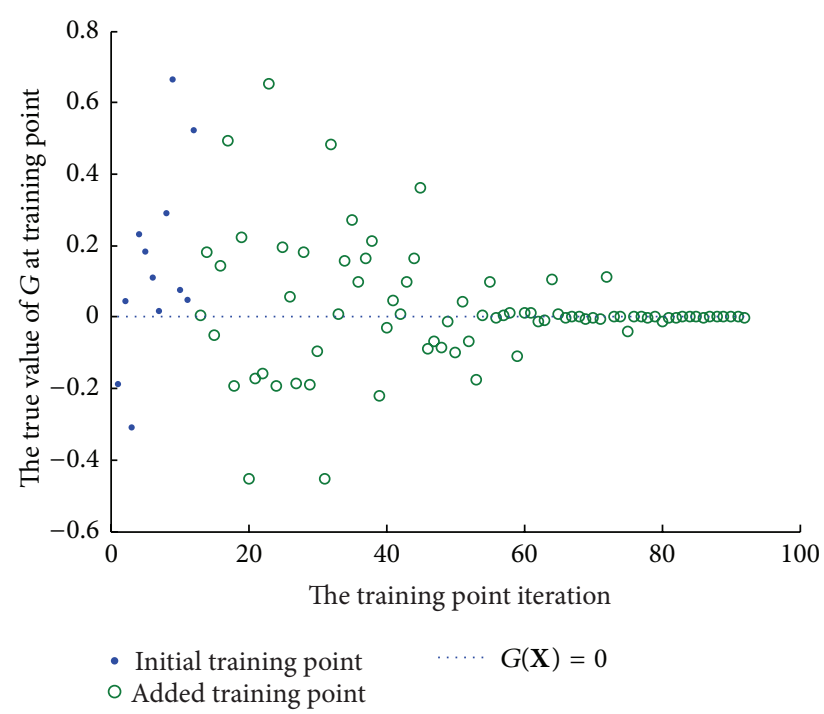

FIGURE 10: Iteration histories of the true value of added training points for 10-bar structure.

that the proposed method is validated to be rational and efficient.

\section{Conflict of Interests}

The authors declare that there is no conflict of interests regarding the publication of this paper.

\section{Acknowledgments}

The financial support from National Natural Science Foundation of China (Grant no. 51205312), The Chinese Aerospace Support Foundation (Grant no. NBXW0001), and The NPU Foundation for Fundamental Research (Grant no. JC20110255) is gratefully acknowledged by the authors.

\section{References}

[1] A. Der Kiureghian, "Analysis of structural reliability under parameter uncertainties," Probabilistic Engineering Mechanics, vol. 23, no. 4, pp. 351-358, 2008.

[2] S. Mahadevan, Probability, Reliability, and Statistical Methods in Engineering Design, John Wiley \& Sons, 2000.

[3] G. I. Schuëller, "On the treatment of uncertainties in structural mechanics and analysis," Computers and Structures, vol. 85, no. 5-6, pp. 235-243, 2007.

[4] P. Bjerager and S. Krenk, "Parametric sensitivity in first order reliability theory," Journal of Engineering Mechanics, vol. 115, no. 7, pp. 1577-1582, 1989.

[5] E. Castillo, R. Mínguez, and C. Castillo, "Sensitivity analysis in optimization and reliability problems," Reliability Engineering and System Safety, vol. 93, no. 12, pp. 1788-1800, 2008.

[6] L. J. Cui, Z. Z. Lü, and X. P. Zhao, "Moment-independent importance measure of basic random variable and its probability density evolution solution," Science China Technological Sciences, vol. 53, no. 4, pp. 1138-1145, 2010.
[7] L. Cui, Z. Lu, and Q. Wang, "Parametric sensitivity analysis of the importance measure," Mechanical Systems and Signal Processing, vol. 28, pp. 482-491, 2012.

[8] M. P. R. Haaker and P. J. T. Verheijen, "Local and global sensitivity analysis for a reactor design with parameter uncertainty," Chemical Engineering Research and Design, vol. 82, no. 5, pp. 591-598, 2004.

[9] A. Saltelli, S. Tarantola, F. Campolongo, and M. Ratto, Sensitivity Analysis in Practice: A Guide to Assessing Scientific Models, John Wiley \& Sons, 2004.

[10] A. Saltelli and J. Marivoet, "Non-parametric statistics in sensitivity analysis for model output: a comparison of selected techniques," Reliability Engineering and System Safety, vol. 28, no. 2, pp. 229-253, 1990.

[11] J. C. Helton and F. J. Davis, "Latin hypercube sampling and the propagation of uncertainty in analyses of complex systems," Reliability Engineering and System Safety, vol. 81, no. 1, pp. 2369, 2003.

[12] H. C. Frey and S. R. Patil, "Identification and review of sensitivity analysis methods," Risk Analysis, vol. 22, no. 3, pp. 553-578, 2002.

[13] H. Rabitz and Ö. F. Aliş, "General foundations of highdimensional model representations," Journal of Mathematical Chemistry, vol. 25, no. 2-3, pp. 197-233, 1999.

[14] A. Saltelli, M. Ratto, T. Andres et al., Global Sensitivity Analysis: The Primer, John Wiley \& Sons, New York, NY, USA, 2008.

[15] I. M. Sobol, "Global sensitivity indices for nonlinear mathematical models and their Monte Carlo estimates," Mathematics and Computers in Simulation, vol. 55, no. 1-3, pp. 271-280, 2001.

[16] W. Yu and T. J. Harris, "Parameter uncertainty effects on variance-based sensitivity analysis," Reliability Engineering \& System Safety, vol. 94, no. 2, pp. 596-603, 2009.

[17] E. Borgonovo, "Measuring uncertainty importance: investigation and comparison of alternative approaches," Risk Analysis, vol. 26, no. 5, pp. 1349-1361, 2006.

[18] E. Borgonovo, W. Castaings, and S. Tarantola, "Moment independent importance measures: new results and analytical test cases," Risk Analysis, vol. 31, no. 3, pp. 404-428, 2011.

[19] Q. Liu and T. Homma, "A new computational method of a moment-independent uncertainty importance measure," Reliability Engineering and System Safety, vol. 94, no. 7, pp. 1205-1211, 2009.

[20] L. Y. Li, Z. Z. Lu, J. Feng, and B. T. Wang, "Moment-independent importance measure of basic variable and its state dependent parameter solution," Structural Safety, vol. 38, pp. 40-47, 2012.

[21] P. Wei, Z. Lu, and X. Yuan, "Monte Carlo simulation for moment-independent sensitivity analysis," Reliability Engineering and System Safety, vol. 110, pp. 60-67, 2013.

[22] D. R. Jones, M. Schonlau, and W. J. Welch, "Efficient global optimization of expensive black-box functions," Journal of Global Optimization, vol. 13, no. 4, pp. 455-492, 1998.

[23] I. Kaymaz, "Application of kriging method to structural reliability problems," Structural Safety, vol. 27, no. 2, pp. 133-151, 2005.

[24] B. J. Bichon, M. S. Eldred, L. P. Swiler, S. Mahadevan, and J. M. McFarland, "Efficient global reliability analysis for nonlinear implicit performance functions," AIAA Journal, vol. 46, no. 10, pp. 2459-2468, 2008.

[25] B. J. Bichon, J. M. McFarland, and S. Mahadevan, "Efficient surrogate models for reliability analysis of systems with multiple failure modes," Reliability Engineering and System Safety, vol. 96, no. 10, pp. 1386-1395, 2011. 
[26] B. Echard, N. Gayton, and M. Lemaire, "AK-MCS: an active learning reliability method combining Kriging and Monte Carlo simulation," Structural Safety, vol. 33, no. 2, pp. 145-154, 2011.

[27] S. N. Lophaven, H. B. Nielsen, and J. Søndergaard, "Dace-a Matlab Kriging Toolbox, Version 2.0,” 2002.

[28] V. Dubourg, B. Sudret, and J.-M. Bourinet, "Reliability-based design optimization using kriging surrogates and subset simulation," Structural and Multidisciplinary Optimization, vol. 44, no. 5, pp. 673-690, 2011.

[29] X. F. Yang, Y. S. Liu, Y. Gao, and Y. S. Zhang, "An active learning Kriging model for hybrid reliability analysis with both random and interval variables," Structural and Multidisciplinary Optimization. In press.

[30] T. Ishigami and T. Homma, "An importance quantification technique in uncertainty analysis for computer models," in Proceedings of the 1st International Symposium on Uncertainty Modeling and Analysis, College Park, Md, USA, December 1990. 


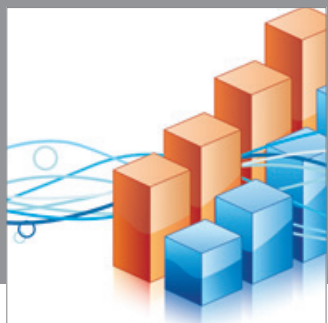

Advances in

Operations Research

mansans

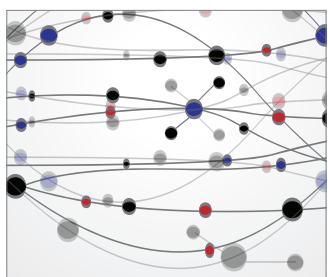

The Scientific World Journal
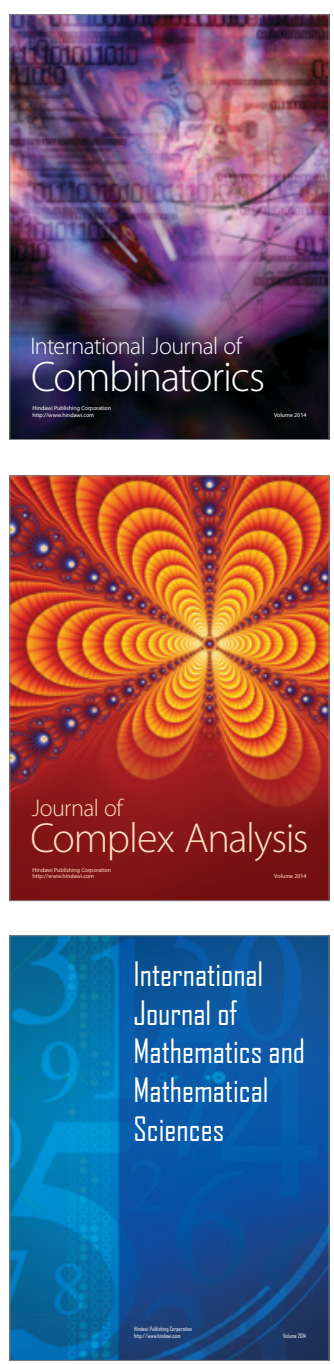
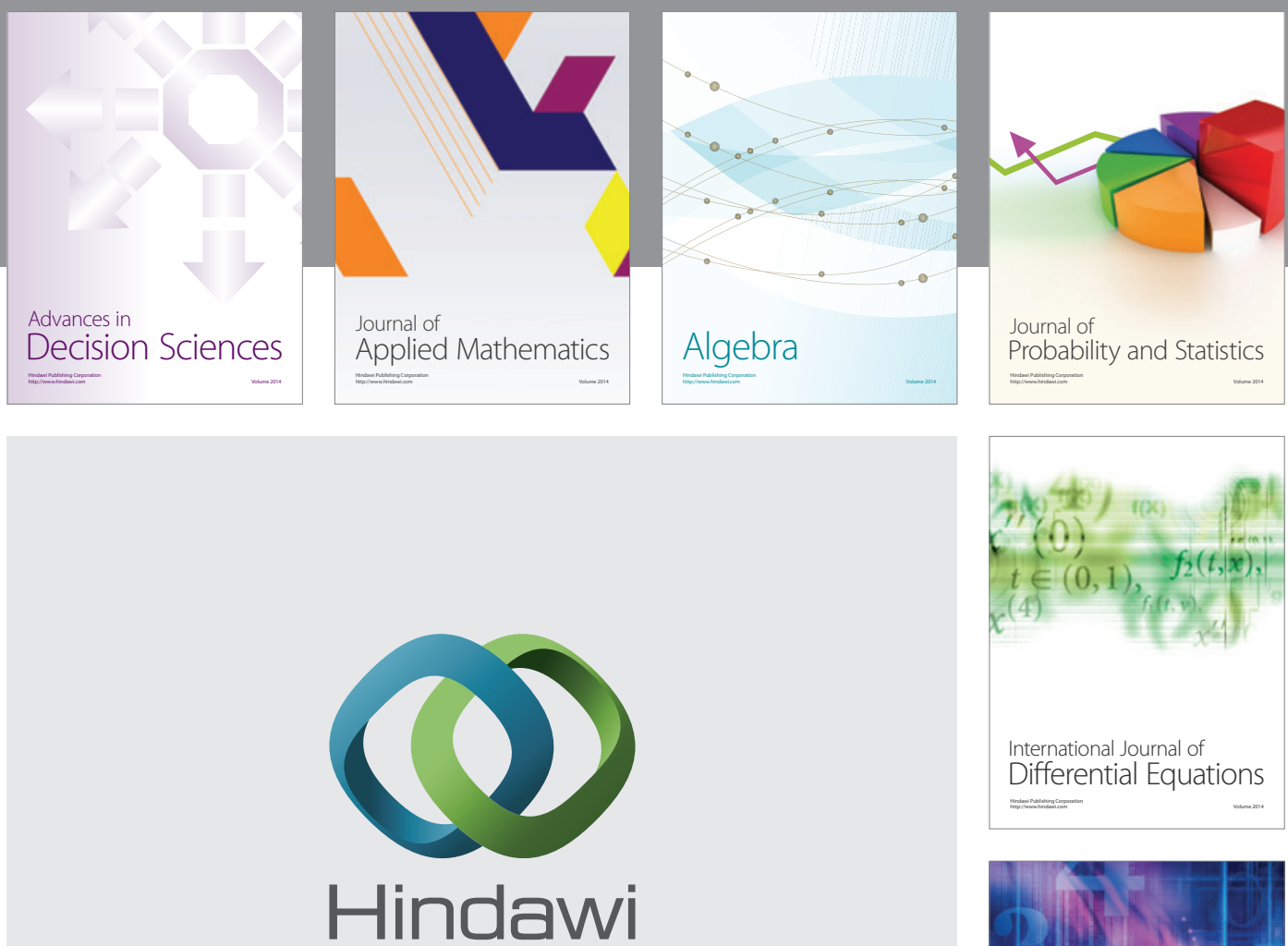

Submit your manuscripts at http://www.hindawi.com
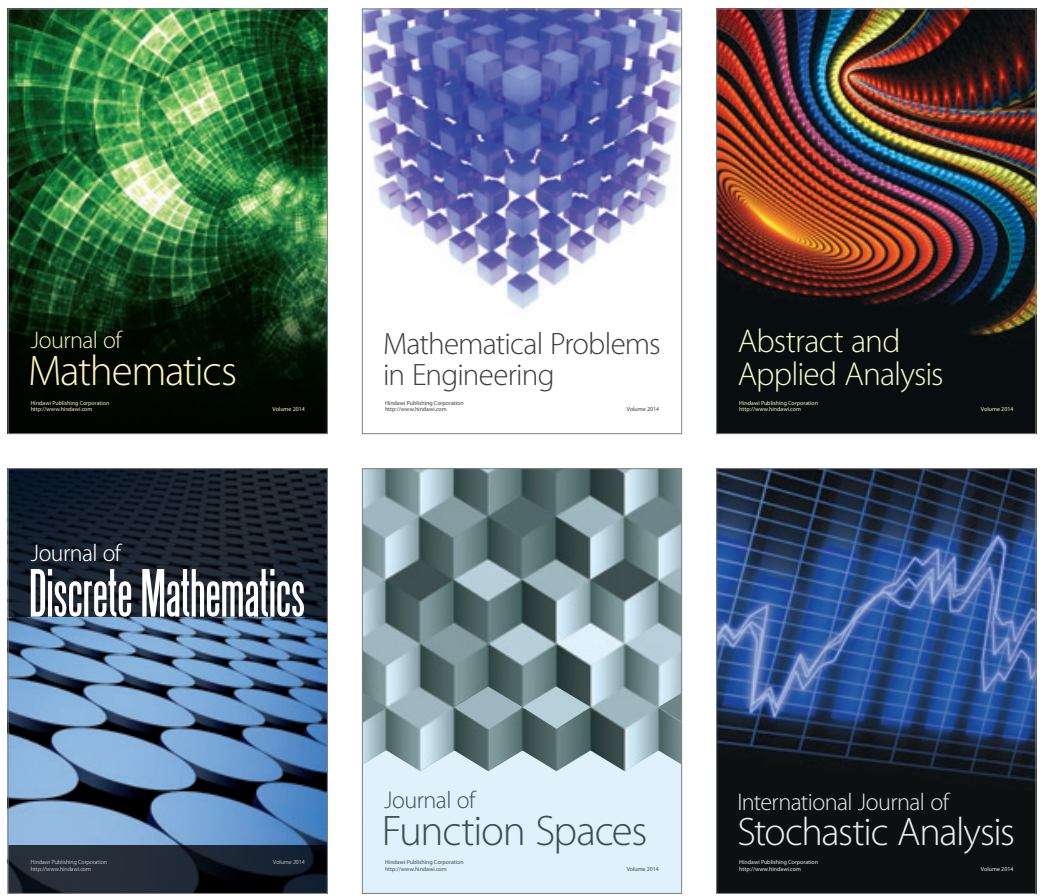

Journal of

Function Spaces

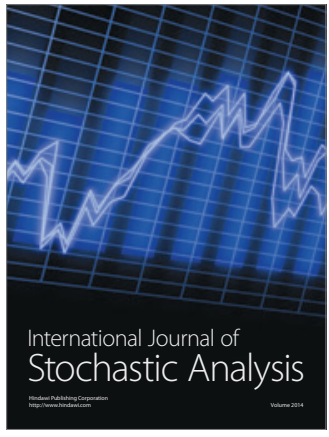

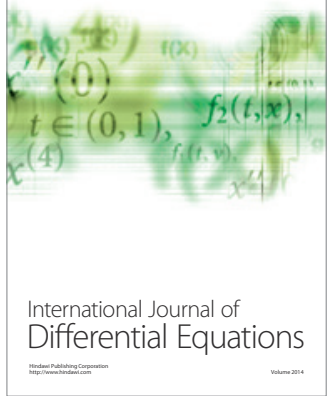
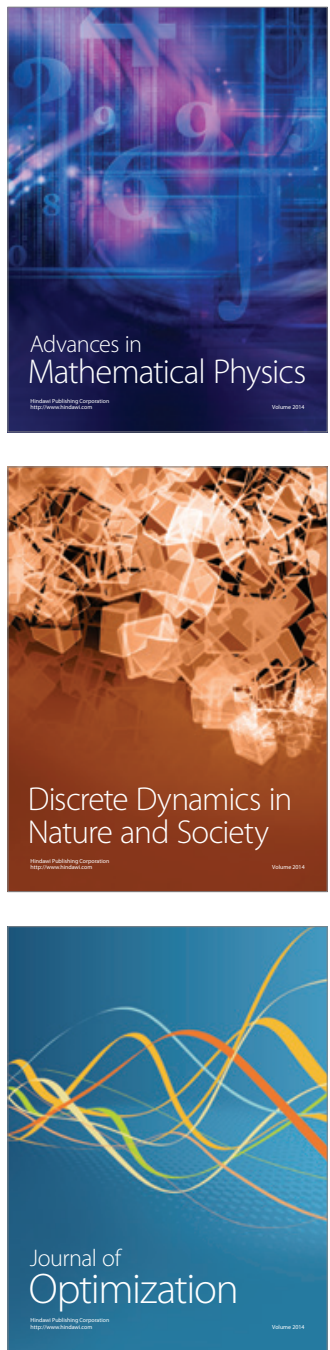